\title{
Need something different? Here's what's been done: Effects of examples and task instructions on creative idea generation
}

\author{
Tim George ${ }^{1} \cdot$ Jennifer Wiley ${ }^{2}$ \\ Published online: 6 January 2020 \\ (C) The Psychonomic Society, Inc. 2020
}

\begin{abstract}
Creative idea generation involves search and retrieval of memory. There is a default tendency to rely too heavily on familiar or easily accessible information during idea generation, especially in tasks such as the alternate uses task (AUT) that involve generating novel uses for common objects. Knowing which obvious ideas to avoid may be important in creating more original ideas. The present experiments tested whether instructions encouraging participants to avoid a set of common example ideas would enhance originality or cause fixation on the AUT. The results of Experiment 1 demonstrated that presenting a verbal list of common example uses along with a warning to avoid those uses enhanced originality. In contrast, when the example ideas were presented in the absence of any "avoid" instructions, there was no benefit on originality, indicating that mere example exposure did not stimulate more creative idea generation. The design of Experiment 2 was parallel to that of Experiment 1, but the verbal examples were replaced with visually depicted examples. Exposure to the visual examples led to reduced originality, suggesting fixation. Although the "avoid" instruction helped to mitigate this fixation, it did not enhance originality beyond the no-example condition. The results suggest that under some conditions presenting unoriginal examples along with an "avoid" warning can allow people to shift their focus away from easily retrieved ideas and toward more novel approaches. The results are also consistent with prior work showing a negative impact of visual presentation of examples on creativity.
\end{abstract}

Keywords Creativity $\cdot$ Fixation $\cdot$ Alternate uses task $\cdot$ Memory $\cdot$ Examples

\section{Introduction}

Creativity and memory are closely linked constructs in that the generation of new ideas involves retrieval of information stored in memory (Madore, Addis, \& Schacter, 2015; Madore, Jing, \& Schacter, 2016; Smith \& Blankenship, 1991; Storm \& Patel, 2014; Ward, Patterson, Sifonis, Dodds, \& Saunders, 2002). We often build off prior experiences and previous ideas when imagining new ideas. Yet, creative thinking involves a difficult balancing act - while new ideas may be built on or inspired by old ones, it also seems important to escape the confines of familiar experiences in order to produce original ideas. For example, the alternate uses task (AUT;

Tim George

georget3@union.edu

1 Union College, Psychology Department, 807 Union St, Schenectady, New York 12308, USA

2 University of Illinois at Chicago, Department of Psychology, 1007 W. Harrison St (MC 285), Chicago, Illinois 60607, USA
Guilford, 1957) represents an instance where the most accessible information in memory is typically the least valuable. In the AUT, a person attempts to think of multiple creative uses for an everyday object, such as a car tire. Performance on this task involves moving past the typical functions of the object (a car part), as well as alternate but well-known ideas (tire swing), so that much more original ideas can be developed (a chandelier).

A diversity of tasks fall under the conceptual umbrella of creative problem solving. Some tasks involve producing multiple ideas in response to an open-ended prompt (e.g., AUT). Others require generating a single non-obvious solution to a problem or puzzle, such as in the remote associates test (RAT; Mednick, 1962) where three problem words (e.g., car, fog, French) are remotely linked to a single solution word $(H O R N)$. Other tasks may involve designing products, or imagining never-before-seen creatures. Although these tasks have different goals, what they have in common is the need to search for and retrieve uncommon associations from memory, while avoiding interference or fixation caused by more common associations (Benedek, Könen, \& Neubauer, 2012; Benedek \& Neubauer, 2013; Maier, 1931; Mednick, 1962). Creative problem-solving tasks involve searching through 
multiple possibilities as well as focusing on single ideas and solutions. In the course of searching through potential ideas, one may be easily constrained by what is familiar or obvious.

Past research on creative problem solving paints a picture in which people do not readily break away from familiar information. When imagining hypothetical extra-terrestrial creatures, it has been found that people heavily draw from the attributes of well-known Earth creatures (Ward et al., 2002) even when instructed to be as different as possible. In more open-ended tasks, such as the AUT, a dominant initial strategy is based on memory-retrieval - people often start off by producing known uses (or ideas closely based on known uses) - and these initial ideas are often the least creative (Beaty \& Silvia, 2012; George \& Wiley, 2019; Gilhooly, Fioratou, Anthony, \& Wynn, 2007; Nusbaum \& Silvia, 2011). This relates to the well-documented serial position effect in tasks intended to measure divergent thinking: earlier ideas tend to be more common, while later ideas are more original (Beaty \& Silvia, 2012; Benedek \& Neubauer, 2013; Christensen, Guilford, \& Wilson, 1957; Hass, 2017; Hass \& Beaty, 2018; George \& Wiley, 2019; Glaveanu, Gillespie, \& Karwowski, 2018; Kraus, Cadle, \& Simon-Dack, 2019; Kudrowitz \& Dippo, 2013). The majority of responses to these idea-generation prompts are typically unoriginal or limited to a restricted set of categories (Beaty \& Silvia, 2012; Ezzat, Agogué, Le Masson, Weil, \& Cassotti, 2018). Although intended to tap imaginative capacity, performance on these idea generation tasks often highlights our default tendency to rely on, or be fixated by, familiar ideas that are easily brought to mind.

The concept of mental fixation dates as least as far as the work of Maier (1931), who illustrated functional fixedness the tendency to consider only common object functions. This can be demonstrated by the two-cord problem. In this problem, the goal is to join together two strings suspended from a ceiling at opposite sides of a room. The strings are too far apart for a person to reach with their arms, but several objects are available in the room, including a pair of pliers. Participants often fail to consider using the pliers as a pendulum weight, which provides an elegant solution for joining the two distantly separated strings. This bias is presumably because only the pliers' typical function is considered by participants. This bias can also be exacerbated by having participants use an object in a typical way immediately before problem solving (Birch \& Rabinowitz, 1951).

Mental fixation has also been experimentally induced in other creative problem-solving tasks such as the RAT. It is easy to become fixated by related, but unviable, associates of the problem words in this task. Moreover, exposure to misleading associates before or during RAT attempts makes solution even less likely by increasing the accessibility of misleading information, which blocks solution (Smith \& Blankenship, 1991; Storm \& Hickman, 2015; Wiley, 1998). Regardless of the type of problem (RAT problems, AUT tasks, design tasks, object- use insight puzzles), an initial search through related associations will take place, during which more immediately activated information is the most likely to impede success (i.e., correct solutions, original ideas). Thus, a commonality between these tasks is that obvious associations can get in the way of more remote information, and prior exposure to obvious associations may further impair performance.

As suggested above, fixation also appears in more open-ended creative problem-solving contexts as exposure to example ideas can negatively impact the generation of subsequent ideas. Much of this work has been done in design contexts, in which exposure to images of devices and products is manipulated prior to idea generation. For instance, Jansson and Smith (1991) exposed engineering students to examples of devices (e.g., a spill-proof coffee mug) prior to generating their own versions of these devices. Compared to a no-exposure control group, participants exposed to examples tended to incorporate design elements of the example devices - even when those devices contained flaws. Constraining effects of flawed example designs have also been obtained using other product design tasks (Cardoso \& Badke-Schaub, 2011; Chrysikou \& Weisberg, 2005). In other work on creative imagination, conformity to examples has been shown in undergraduate students tasked with drawing new designs for toys and alien creatures (Marsh, Landau, \& Hicks, 1996; Smith, Ward, \& Schumacher, 1993). Prior to generating ideas, half of the participants viewed examples of toys or creatures that all contained three prototypical features (examples condition). Compared to a control condition that viewed no examples, participants in the examples condition incorporated more of the prototypical features into their designs - that is, their ideas conformed to the examples. Negative effects of example ideas have also been demonstrated in group brainstorming about improvement prompts (e.g., improving a campus), in which exposure to ideas occurs via group members, which impairs idea production (Kohn \& Smith, 2011). More recent work has demonstrated that exposure to common noun-verb pairs (e.g., shoe-walk) can constrain participants' subsequent creativity when later prompted to generate to creative verbs in response to those nouns (e.g., shoe-?; Beaty, Christensen, Benedek, Silvia, \& Schacter, 2017).

While these findings suggest that example exposure can impair creativity, other results indicate that the relationship is more complex. For example, while presenting common examples can restrict creativity, presenting unusual examples can enhance creativity relative to control conditions (Agogué et al., 2014; George, Wiley, Koppel, \& Storm, 2019; Sio, Kotovsky, \& Cagan, 2015; Yagolkovskiy \& Kharkhurin, 2016). Mixed findings have also been obtained with respect to instructions given to participants regarding examples. Jansson and Smith (1991) and Smith et al. (1993) found that participants conformed to examples even when explicitly instructed that they should avoid features of the examples. This aligns with a fixation account in which the scope of ideas generated is not affected so much by the appraisal of 
information as the mere presence of that information. On the other hand, Chrysikou and Weisberg (2005) found that "defixating" instructions aimed at getting participants to avoid problematic aspects of examples were effective in reducing participants' conformity on a design task. In these experiments, participants viewed example products that contained specific problematic elements, such as a disposable coffee cup that would easily spill when squeezed. Compared to the control group (no examples), participants who were not instructed to avoid these problematic elements produced designs with more similarity to the examples, while participants who were instructed to avoid them (de-fixation) produced designs that were less similar to the examples. However, the similarity of the designs in the de-fixation and control conditions did not differ (i.e., there was no added benefit of de-fixation in terms of dissimilarity of designs). Additionally, this study did not use a measure of originality or creativity. More recently, Ezzat et al. (2018) found that instructing people to avoid restrictive ideas improved originality on a task involving devising solutions for dropping an egg from a building without breaking the egg. Using the same task, when participants were instructed to "search for another path" whenever they produced an idea from a common category, this resulted in fewer typical ideas (Ezzat et al., 2017). Another recent study has demonstrated that showing people unfunny examples of humor to be avoided increased the quality of participants' jokes relative to a no-examples group (Shin, Cotter, Christensen, \& Silvia, 2018), indicating that sometimes creative performance can benefit from being directed to avoid specific obvious ideas. However, this set of studies on examples and de-fixation did not include conditions in which people were generally instructed to avoid problematic ideas without reference to any examples, making it somewhat difficult to determine if any mitigating effects of de-fixation instructions are due to the examples, instructions, or a combination of the two.

Other work has attempted to simply encourage people to be creative without reference to any specific examples. Some studies have demonstrated that explicitly prompting people to "be creative" when coming up with ideas results in higher originality than simply prompting people to list ideas (Chen et al., 2005; Forthmann et al., 2016; Goldenberg, Larson, \& Wiley, 2013; Harrington, 1975; Nusbaum, Silvia, \& Beaty 2014; Ward et al., 2002). However, it should be noted that emphasizing creativity still tends to result in a majority of responses that are unoriginal. Even when instructed to be as "wild" or as different as possible (Goldenberg et al., 2013; Manske \& Davis, 1968; Osborn, 1953), people still tend to preserve the properties of known examples.

Taken together, this overall body of work on examples and instructions suggests that it may be possible to push people to be more original, and that such direction is important for getting people away from a default tendency to simply list known ideas rather than create new ones.

\section{Current investigation}

Common associates exert a strong influence on creative idea generation. An important aim of the present research is to explore how the manner in which creative idea generation tasks are constructed may moderate this influence. Specifically, because the goal in these tasks is to be original, one possibility is that before setting out to produce ideas, it may be important for people to have a sense of which ideas are common and unoriginal, so that more interesting and novel approaches can be considered. This goal was explored in two ways: instructing participants to avoid unoriginal ideas, and exposing them to examples of unoriginal ideas. The AUT was chosen because it is a commonly used task in creativity investigations, and because of the high chances of becoming stuck on highly available ideas (i.e., mentally fixated) - a common occurrence in real-world creative-thinking scenarios. Additionally, one can identify a clear set of frequently generated responses - making it ideal for exploring potential fixation from example exposure. Yet, surprisingly little research has systematically assessed how example exposure might influence fixation or originality in performance on the AUT.

Additionally, some prior work has shown that fixation on the AUT can be affected by presenting the target object that one is prompted to generate alternate uses for in a visual rather than a verbal form (e.g., a line drawing of a brick vs. displaying the word "brick"). Visual object prompts have been shown to elicit responses more closely related to an object's typical action (Chrysikou, Motyka, Nigro, Yang, \& Thompson-Schill, 2016). The current study extends this prior work by investigating verbal versus visual depiction when exposing participants to example uses, exploring how that exposure will impact originality, and whether an instruction can mitigate against any negative effects.

There are two contrasting accounts for how instructing people to avoid unoriginal ideas and exposing them to examples will impact creative idea generation.

Fixation account It could be argued that any sort of attention to common ideas could be detrimental for creative idea generation and cause fixation - even when instructed to avoid those ideas. The AUT in particular involves salient familiar ideas exerting a strong influence over the generation of new ideas (Gilhooly et al., 2007). When brainstorming about new uses for objects, a set of common ideas stored in memory may be easily and automatically brought to the forefront of one's attention, at the expense of creating other potentially more unusual ideas. The ease with which such ideas come to mind may further lead people to adopt a "path of least resistance" approach to idea production (Ward et al., 2002). Exposure to examples, even with instructions to avoid these examples, may induce an ironic process in which thoughts or themes to be avoided nonetheless pop into mind (e.g., white bears - 
Wegner, 1994; or baseball - Wiley, 1998). Additionally, memory research in part-set cuing (Rhodes \& Castel, 2008) indicates that cuing people with a subset of previously learned items impairs recall of the remaining set relative to freerecall conditions, and this occurs in both episodic and semantic tasks. In this sense, the example ideas may act in a similar manner to this subset which impairs the creation of new ideas.

Direction account On the other hand, without providing people with a clear sense of ideas that are unoriginal, people may fail to readily discard obvious ideas when brainstorming. In the AUT, perhaps by not only showing people unoriginal example ideas but framing them as unoriginal, this may push people to think more carefully about their own ideas. For example, when prompted to think of uses for a car tire, encouraging people to think beyond ideas like "a tire swing" or "exercise equipment" may force them to reconsider their default approach, and shift them into more strategic or controlled memory processes. A set of common ideas may further serve as a reference point by which people could compare potential new ideas against more common ones. This suggests a "direction" account in which knowing what to avoid is important, which makes the prediction that when presented with a set of common example ideas and with instructions encouraging people to avoid a set of common example ideas, this will result in a greater amount of original ideas in the AUT. This contrasts with the fixation account (Jansson \& Smith, 1991; Kohn \& Smith, 2011; Smith et al., 1993), which makes the alternate prediction that any example exposure will constrain the accessibility of ideas, leading to reduced originality.

In Experiment 1, the set of common examples were presented in verbal format. Because it has been argued that visual examples are especially likely to bias people toward typical functions (Camarda et al., 2018; Chrysikou et al., 2016), the second experiment used visual examples to test whether fixating/facilitating effects of examples might depend on presentation modality.

\section{Experiment 1}

The primary goal of Experiment 1 was to test whether providing instructions encouraging people to avoid a list of examples of commonly generated, unoriginal ideas (Examples+Avoid), would help improve originality in the AUT, or if such a manipulation would lead to mental fixation. All participants generated uses for a car tire. Performance in the Examples+Avoid condition was compared to performance in three other conditions. One comparison condition received neither the instruction to avoid a list of examples of commonly generated uses, nor the list of examples (NoExamples+NoAvoid). A second comparison condition included a list of common example ideas with no instruction to avoid such ideas (Examples+
NoAvoid). This was intended to test among potential alternate explanations. First, simply the presence of example ideas may have a stimulating effect on originality, even without instructions to avoid them. If the example ideas, although unoriginal, help inspire participant's own original ideas, then one might expect a benefit of example exposure even when avoid instructions are not present. Alternatively, another possibility is that fixating effects of common examples will emerge when there are no instructions encouraging people to avoid such examples, which will reduce originality relative to the NoExamples+NoAvoid condition.

Additionally, any manipulation encouraging people to avoid a list of common ideas contains an implicit hint that ideas that many other people think of are unoriginal and should be avoided. In this sense, any observed benefit of the Examples+Avoid condition may be due to the general additional "push" to avoid obvious ideas, that is not tied to any specific list of examples. Therefore, a fourth comparison condition included instructions to avoid ideas that many other people might think of without reference to any specific list of common examples (NoExamples+Avoid). To test the hypothesis that the Examples+Avoid condition would result in the highest originality, a planned comparison between this group and all other conditions was conducted.

This experiment also explored how the originality of ideas changes from earlier ideas to later ideas. By examining how originality changes across the series of ideas that are generated, this can help further clarify any potential benefits or decrements of example exposure. Although people frequently experience mental fixation when attempting to generate ideas, it also appears that people can often overcome this fixation and eventually improve the novelty of their ideas over the course of idea generation (Beaty \& Silvia, 2012; Benedek \& Neubauer, 2013; Christensen et al., 1957; Hass, 2017; Hass \& Beaty, 2018; George \& Wiley, 2019; Glaveanu et al., 2018; Kraus et al., 2019; Kudrowitz \& Dippo, 2013). One way of viewing any lack of improvement in originality from earlier to later ideas (i.e., no serial position effect) may be as a form of continued fixation. On the other hand, one potential consequence of instructions to avoid common ideas may be that people bypass unoriginal ideas early on and continue to generate original ideas throughout the task, which would result in a reduced serial position effect.

\section{Method}

Participants and design Participants were 144 undergraduates (97 female, $M_{\text {age }}=18.73$ years, $S D=1.68$ ) from the University of Illinois at Chicago psychology subject pool. Based on the study by Shin et al. (2018) who found that better jokes were produced by a group exposed to unfunny examples than no examples $(d=.85)$, a power analysis revealed this sample should be sufficient to detect a similar effect with 
$94 \%$ power. This power analysis was used to determine the sample size for both studies. All participants provided agreement to participate and were compensated with course credit. The design was a $2 \times 2$ between-subjects design crossing Example Exposure (examples vs. no examples) and Instruction (avoid vs. no avoid) with 36 participants randomly assigned to each condition.

Materials The prompt for the AUT was the car tire item. This item was selected because prior work in the same population found that there was a clear set of five commonly occurring ideas in response to this prompt (George \& Wiley, 2019): (1) Tie a rope to it and make a tire swing out of it, (2) Use it as a chair to sit in, (3) Use it as an exercise tool by lifting it up and down, (4) Get inside it and roll down a hill, (5) Plant flowers in it. These served as examples of unoriginal ideas in the two examples conditions.

Procedure Most tasks were completed via computer. All participants were instructed that they would be thinking of creative uses for a car tire. Participants were told that there are many common, unoriginal ways to use a car tire, but that for this task they should generate all of the unusual, creative, and uncommon uses they can think of. The exact instructions for the remaining conditions are shown in Appendix 1. In the Examples+ Avoid condition, an additional instruction screen presented the five examples along with instructions to avoid those ideas.

In the Examples+NoAvoid condition, the examples were presented simply as example ideas with no instruction to avoid them. Additionally, during the idea generation phase, the phrase "unoriginal ideas" above the list of example ideas was replaced with "example ideas."

In the NoExamples+Avoid condition, participants were instructed that they should try to avoid unoriginal ideas that others might think of, without providing any specific examples.

All participants were reminded to be as creative as possible before beginning the idea-generation phase. For the ideageneration phase, participants had 3 min to generate ideas. The name of the item ("Car tire") remained in the upper left corner of the screen. In the examples conditions, the five example ideas remained on the left side of the screen during idea generation. They indicated when they had an idea in mind by pressing the space bar, after which a text box appeared on the right side of the screen and participants typed their ideas. The computer logged response time and typing time. After entering each idea, they were prompted to continue generating ideas until the time was up.

Then, participants completed a post-AUT questionnaire. Using a 1-5 agreement scale, participants rated their perceptions of the task in the following order: (1) the difficulty of generating ideas, (2) how stuck they felt, (3) how stuck they felt on obvious ideas, (4) how much their earlier ideas got in the way of their later ideas, (5) whether their ideas were of above-average creativity, (6) the extent to which they generated familiar ideas they knew about prior to the experiment, and (7) the extent to which they generated brand new ideas they had not thought of previously. Participants in the examples conditions were asked an additional set of questions about the examples. They were asked to rate (8) how much the example ideas got in the way of generating their own ideas, (9) how distracting the examples were, (10) how helpful the example ideas were, (11) how much the examples helped them avoid obvious ideas, and (12) how much they built their own ideas off the example ideas. Following the questionnaire, participants in the examples conditions were asked to recall all five example ideas on a sheet of paper.

Coding Several behavioral measures were computed in relation to the AUT. First, the number of ideas generated was assessed by removing redundant and incomplete/incoherent responses, and identifying ideas that matched the examples. Two raters coded a random subset of $25 \%$ of responses. Reliability (consistency of ratings) was excellent, $\operatorname{ICC}(2,2)=$ $.98,95 \%$ CI $[.97, .99]$. The first rater coded the remaining responses. This resulted in three counts: (1) Total fluency (the total number of ideas a participant generated), (2) number of example ideas generated, and (3) non-example fluency (the total number of non-example ideas a participant generated).

Originality of non-example ideas (the primary dependent measure) was assessed with a frequency-based method, in which the proportion of people giving each response was calculated. Responses occurring $1 \%$ of the time or less within the corpus of all responses were categorized as "original" ideas while those occurring greater than $1 \%$ of the time were not. From this initial coding, two commonly used alternate scores were derived: one summative measure and one ratio measure. For each participant, the summative measure was simply the number of original (non-example) ideas generated (Agogue et al., 2014; Radel, Davranche, Fournier, \& Dietrich, 2015). A second ratio measure divided the number of original (nonexample) responses by the total of (non-example) responses (Forthmann, Lips, Szardenings, Scharfen, \& Holling, 2018; Forthmann, Szardenings, \& Holling, 2018). Analyses of these two measures yielded similar patterns, as reported below.

Flexibility, or the number of categories of ideas that a person spanned in their set of responses, was also calculated. Responses were grouped into 16 broad semantic categories. Responses that shared similar semantic themes were grouped into the same category (see Appendix 2). Two raters coded a random subset of $25 \%$ of responses. Reliability (consistency of ratings) was excellent $\operatorname{ICC}(2,2)$ $=.91,95 \% \mathrm{CI}[.86, .94]$. The first rater coded the remaining responses into categories. To provide a measure that was fair across conditions, flexibility was calculated by computing the sum of categories after excluding any example ideas from the participants' responses. 
Mean idea response time was calculated from the total time (s) spent generating each idea. This included both time until button press indicating that an idea was thought of, and time spent typing the idea. The reason for including typing time in the response time measure is that inspection of typing times along with anecdotal reports indicated that many participants pressed the idea button even when an idea was not fully articulated (e.g., a person taking $37 \mathrm{~s}$ to type the phrase "flower pot"). Thus, this total time measure provides a slightly more realistic measure of the amount of time spent thinking about each idea. The second timing measure that was calculated was the time stamp following the last example idea that was generated.

Idea position was also logged for analysis of serial order. The use of idea position for analysis of changes in originality over the course of idea generation is common (Christensen et al., 1957; Benedek \& Neubauer, 2013; George \& Wiley, 2019; Glaveanu et al., 2018; Hass, 2017; Hass \& Beaty, 2018; Kraus et al., 2019; Kudrowitz \& Dippo, 2013). Although cumulative response time could be used to analyze how ideas change over time, the issues with the accuracy of RT logging mentioned above make this an unsuitable approach. Nonetheless, idea position can be used for a meaningful analysis of serial order because it is not just the passage of time that produces the serial order effect, but the production of ideas - people start off by generating many common ideas, and then need to shift to more original ones.

Recall protocols were scored for the number of examples that were correctly recalled. However, it should be noted that due to a procedural error, only 14 participants in the Examples+Avoid condition were asked to recall the examples. Two raters coded a random subset of $25 \%$ of protocols. Reliability was excellent $\operatorname{ICC}(2,2)=.98,95 \%$ CI $[.97, .99]$. The first rater coded the remaining protocols. Example recall, the percentage of example ideas correctly recalled, was calculated for participants in the examples conditions.

\section{Results}

Number of original responses (originality) ${ }^{1}$ For summative originality scores, there was a main effect of Example Exposure, $F(1,140)=4.85, p=.030, \eta_{\mathrm{p}}{ }^{2}=.03$, and a main effect of Avoid Instruction, $F(1,140)=5.60, p=.019, \eta_{\mathrm{p}}{ }^{2}=$ .04 . On average, the groups receiving examples generated more original ideas $(M=2.65, S D=2.49)$ than those not

\footnotetext{
${ }^{1}$ For the ratio score, there was no main effect of Example Exposure, $F(1,140)$ $=0.79, p=.375, \eta_{\mathrm{p}}{ }^{2}=.01$, a marginal main effect of Avoid Instruction, $F(1$, $140)=3.41, p=.063, \eta_{\mathrm{p}}{ }^{2}=.02$, with a greater originality proportion in the Avoid $(M=.53, S D=.30)$ than No-Avoid conditions $(M=.44, S D=.28)$ and a marginal interaction, $F(1,140)=3.26, p=.073, \eta_{\mathrm{p}}^{2}=.02$. For the planned comparison, the Examples+Avoid group generated a greater proportion of original ideas $(M=.60, S D=.28)$ than all the others $(M=.45, S D=.29)$, $t(142)=2.65, p=.009, d=0.51$.
}

receiving examples $(M=1.90, S D=1.58)$. On average, the groups receiving avoid instructions generated more original ideas $(M=2.68, S D=2.51)$ than those not receiving avoid instructions $(M=1.88, S D=1.53)$. The interaction did not reach significance, $F(1,140)=2.16, p=.14, \eta_{\mathrm{p}}^{2}=.02$. However, as shown in Fig. 1, the planned comparison showed that the Examples+Avoid group generated the greatest number of original ideas compared to all other groups, $t(142)=3.51, p$ $=.001, d=0.58$. The other conditions did not significantly differ from each other.

Originality across idea position Additionally, to explore how the likelihood of original responses changes over the course of idea generation, effects of idea position on originality were calculated. The order of each participant's ideas was logged. Next, mixed-effects logistic models predicting originality were run for each condition using the lme4 package (Bates, Maechler, \& Bolker, 2012) in R. Idea position was used as the fixed effect. As random effects, the by-subject random intercepts and random slopes for the effect of idea position were used. As shown in Fig. 2, there were increases in the likelihood of an original idea across position in all four conditions: NoExamples+NoAvoid condition $(\beta=0.18, S E=0.06, Z=$ $2.91, p=.004)$; NoExamples+Avoid condition $(\beta=0.20, S E$ $=0.07, Z=2.94, p=.003)$; Examples+Avoid condition $(\beta=$ $0.21, S E=0.07, Z=3.14, p=.002)$; however, the effect was marginal in the Examples+NoAvoid condition $(\beta=0.14, S E=$ $0.07, Z=1.87, p=.061)$.

Other AUT measures The means across conditions for the other dependent measures are displayed in Table 1.

Fluency measures For total (summative) fluency, there were no significant effects for Example Exposure, $F(1,140)=0.50$, $p=.481, \eta_{\mathrm{p}}^{2}=.004$, Avoid Instructions, $F(1,140)=0.00$, the interaction, $F(1,140)=0.41, p=.522, \eta_{\mathrm{p}}^{2}=.003$, nor the planned comparison, $t(142)=0.97, p=.970, d=0.01$.

For the number of example responses (summative), ${ }^{2}$ there was a main effect of Example Exposure, $F(1,140)$ $=37.06, p<.001, \eta_{\mathrm{p}}{ }^{2}=.21$, such that groups receiving no example ideas spontaneously produced more of the example ideas $(M=1.77, S D=1.20)$ than the examples

\footnotetext{
${ }^{2}$ A ratio score for the number of example responses (number of example responses divided by total responses) was also computed. This yielded a main effect of Example Exposure, $F(1,140)=44.09, p<.001, \eta_{\mathrm{p}}{ }^{2}=.24$ with a greater proportion of example responses (and relatively lower proportion of non-example responses) in the No Examples conditions $(M=.32, S D=.19)$ than No Examples conditions $(M=.12, S D=.19)$, a main effect of Avoid Condition, $F(1,140)=9.44, p=.003, \eta_{\mathrm{p}}^{2}=.06$, with a greater proportion of example responses (and relatively lower proportion of non-example responses) in the No Avoid $(M=.27, S D=.22)$ than Avoid condition $(M=$ $.17, S D=.20)$ and no interaction, $F(1,140)=0.56, p=.454, \eta_{\mathrm{p}}{ }^{2}=.004$. For the planned comparison, the Examples+Avoid group generated a smaller proportion of example responses $(M=.27, S D=.21)$ than the other conditions $(M=$ $.06, S D=.14), t(142)=5.74, p<.001, d=1.17$.
} 


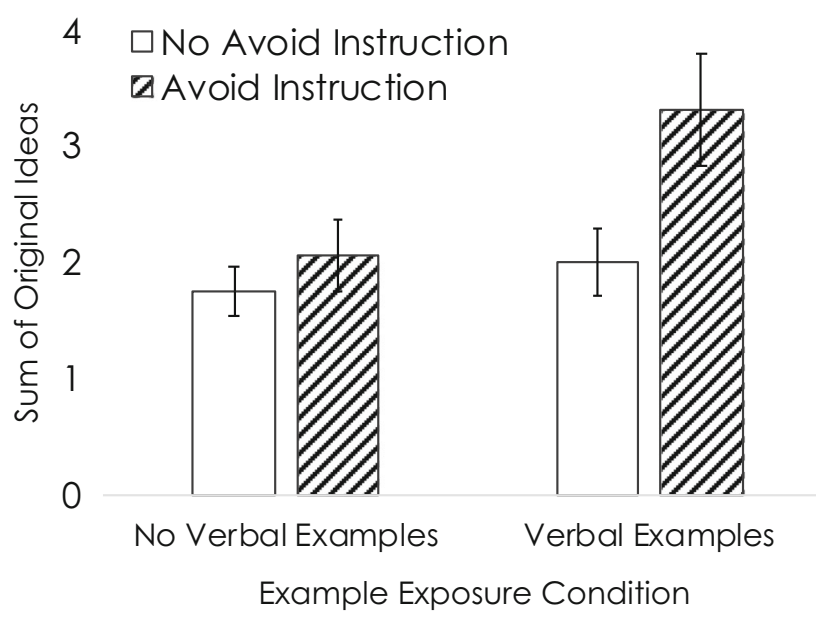

Fig. 1 Mean number of original ideas by condition in Experiment 1 . Error bars represent standard errors (1 SEM)

groups $(M=0.65, S D=1.06)$. There was also a main effect of Avoid Instruction such that the groups
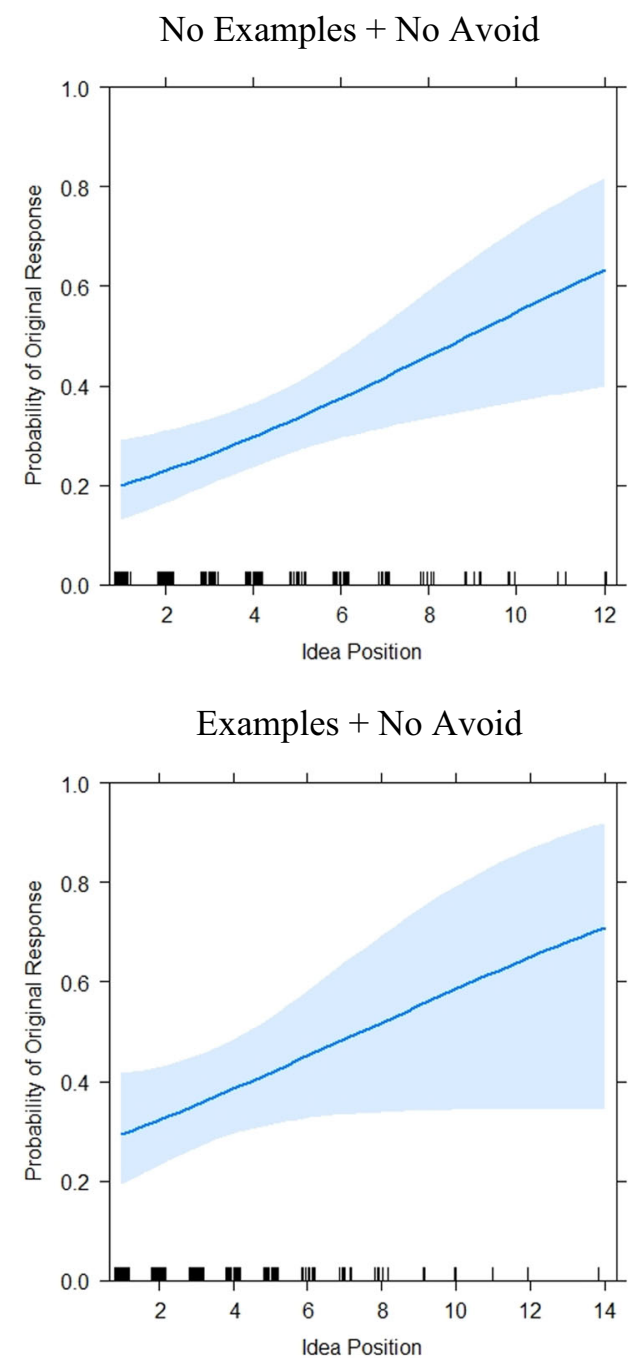

receiving avoid instructions generally generated fewer examples ideas $(M=.94, S D=1.13)$ than groups without the avoid instructions $(M=1.49, S D=1.33), F(1$, 140) $=8.59, p=.004, \eta_{\mathrm{p}}^{2}=.06$. This indicates that these five example ideas are likely to be spontaneously generated under normal conditions, and that participants in the examples conditions largely avoided them. The interaction was not significant, $F(1,140)=0.05, p=$ $.822, \eta_{\mathrm{p}}^{2}=.000$. For the planned comparison, the Examples+Avoid group generated fewer example ideas $(M=.36, S D=.80)$ than all other conditions $(M=$ $1.50, S D=1.26), t(142)=5.07, p<.001, d=1.08$.

For non-example fluency (summative), there was a main effect of Example Exposure, $F(1,140)=4.52, p=.035$, $\eta_{\mathrm{p}}{ }^{2}=.03$, with the example groups generally generating more non-example ideas $(M=4.65, S D=2.62)$ than the no-examples groups $(M=3.83, \mathrm{SD}=1.97)$. There was no significant effect of Avoid Instruction, $F(1,140)=1.98$, $p=.16, \eta_{\mathrm{p}}^{2}=.01$, and the interaction was not significant,
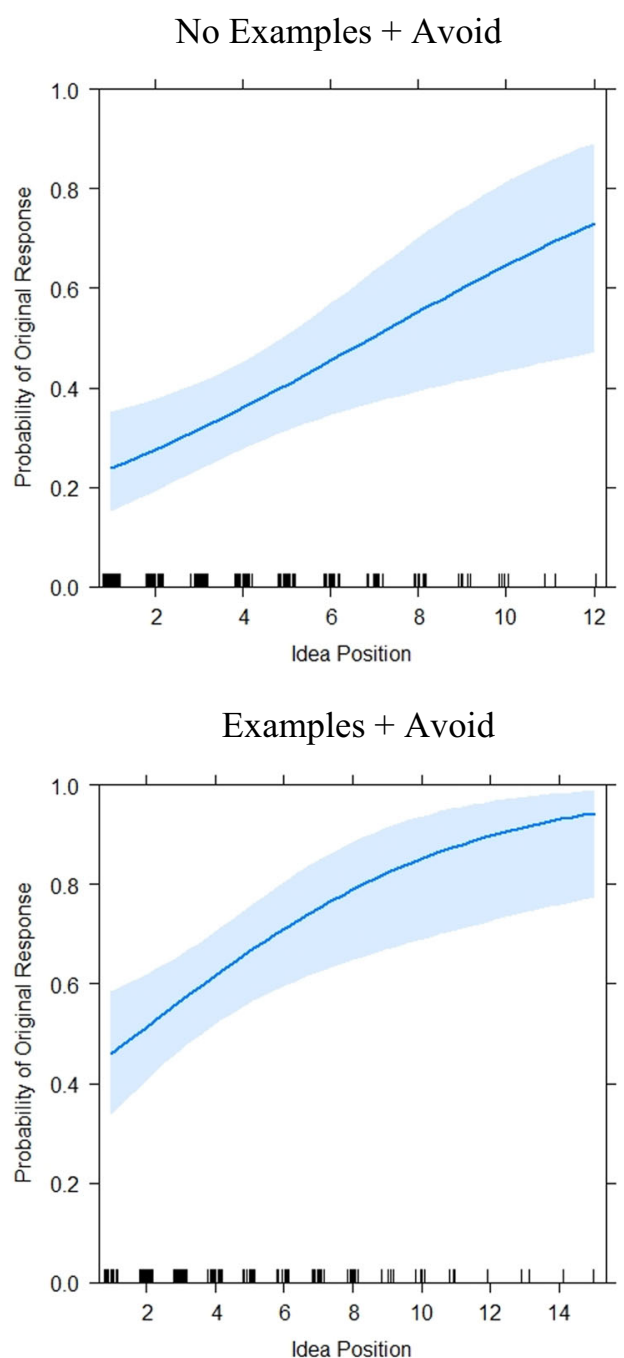

Fig. 2 Effects of idea position on likelihood of original responses across warning and verbal example conditions in Experiment 1. Shaded areas indicate $95 \%$ confidence bands 
Table 1 Mean values for AUT measures by condition in Experiment 1

\begin{tabular}{lcccc}
\hline & \multicolumn{1}{l}{$\begin{array}{l}\text { NoExamples+NoAvoid } \\
M(S D)\end{array}$} & $\begin{array}{l}\text { Examples+NoAvoid } \\
M(S D)\end{array}$ & $\begin{array}{l}\text { NoExamples+Avoid } \\
M(S D)\end{array}$ & $\begin{array}{l}\text { Examples+ Avoid } \\
M(S D)\end{array}$ \\
\hline Fluency (Total) & $5.75(2.25)$ & $5.17(2.35)$ & $5.47(2.50)$ & $5.44(3.18)$ \\
Fluency (Example responses) & $2.03(1.23)$ & $0.94(1.22)$ & $1.53(1.13)$ & $0.36(0.80)$ \\
Fluency (Non-example responses) & $3.72(1.84)$ & $4.22(2.07)$ & $3.94(2.91)$ & $5.08(3.04)$ \\
Flexibility (Non-example responses) & $3.11(1.41)$ & $3.42(1.46)$ & $2.83(1.38)$ & $3.89(2.06)$ \\
Average Response Time per Idea (s) & $37.15(24.72)$ & $42.93(23.02)$ & $36.27(19.48)$ & $43.27(23.22)$ \\
Last Example Response Time Stamp (s) & $106.17(57.63)$ & $93.87(55.87)$ & $74.65(52.01)$ & $72.66(32.25)$ \\
Example Recall (Proportion) & - & $.73(.27)$ & - & $.73(.26)$ \\
\hline
\end{tabular}

$F(1,140)=0.69, p=.41, \eta_{\mathrm{p}}{ }^{2}=.01$. For the planned comparison, the Examples+Avoid group generated more nonexample ideas $(M=5.08, S D=3.03)$ than all other conditions $(M=3.96, S D=2.00), t(142)=2.53, p=.013, d=0.43$.

Flexibility $^{3}$ For flexibility (summative number of categories computed excluding examples), there was a main effect of Example Exposure, $F(1,140)=6.47, p=.012, \eta_{\mathrm{p}}{ }^{2}=.04$, with the examples groups showing higher flexibility $(M=3.65, S D$ $=1.79)$ than the no-examples groups $(M=2.97, S D=1.39)$. There was no significant main effect of Avoid Instruction, $F(1$, $140)=0.13, p=.717, \eta_{\mathrm{p}}{ }^{2}=.00$. The interaction was not significant, $F(1,140)=1.96, p=.163, \eta_{\mathrm{p}}{ }^{2}=.01$. For the planned comparison, the Examples+Avoid group showed higher flexibility $(M=3.89, S D=2.07)$ than all other conditions $(M=3.15, S D=1.42), t(142)=2.48, p=.014, d=0.42$.

Average response time per idea For idea response time, there was a main effect of Example Exposure, $F(1,140)=2.86, p=$ $.093, \eta_{\mathrm{p}}{ }^{2}=.02$, with marginally longer response times in the examples groups $(M=43.1 \mathrm{~s}, S D=23.0 \mathrm{~s})$ than the noexamples groups $(M=36.7 \mathrm{~s} S D=22.1 \mathrm{~s})$. There was no main effect of Avoid Instruction, $F(1,140)=0.01, p=.940, \eta_{\mathrm{p}}{ }^{2}=$ .00 . For the planned comparison of Examples+Avoid and all other conditions, there was no significant difference, $t(142)=$ $1.03, p=.305, d=0.19$.

In order to provide some estimate of how long participants may have spent generating the example ideas (particularly those in the no-examples conditions), the second timing measure that was calculated was the time stamp following the last example idea that was entered by each participant. There was no main effect of Example Exposure, $F(1,84)=0.29$,

\footnotetext{
${ }^{3}$ As with originality, a ratio score for flexibility was also computed. This yielded no main effect of Example Exposure $F(1,140)=0.12, p=.732, \eta_{\mathrm{p}}{ }^{2}$ $=.001$, a main effect of Avoid Instruction $F(1,140)=3.94, p=.049, \eta_{\mathrm{p}}{ }^{2}=.03$, with a greater flexibility proportion in the No Avoid $(M=.86, S D=.20)$ than Avoid condition $(M=.79, S D=.19)$ and no interaction, $F(1,140)=1.45, p=$ $.231, \eta_{\mathrm{p}}{ }^{2}=.01$, and no difference in the planned comparison $t(142)=0.64, p=$ $.521, d=0.13$.
}

$p=.592, \eta_{\mathrm{p}}{ }^{2}=.00$, a marginal main effect of Avoid Instruction, $F(1,84)=3.94, p=.050, \eta_{\mathrm{p}}{ }^{2}=.05$, with a later time stamp in the No Avoid $(M=101.5 \mathrm{~s}, S D=56.7 \mathrm{~s})$ than the Avoid conditions $(M=74.2 \mathrm{~s}, S D=48.7 \mathrm{~s})$, and no interaction, $F(1,84)=0.15, p=.699, \eta_{\mathrm{p}}{ }^{2}=.00$. For the planned comparison of Examples+Avoid and all other conditions, there was no significant difference, $t(86)=0.92, p=.359, d=0.39$.

Post-AUT questionnaire The means for the responses to the post-task questionnaire are shown in Table 2. For the questions that were asked across all participants, the only ANOVA that reached significance was the rating of the degree to which participants felt they generated familiar ideas. There was a main effect of Example Exposure, $F(1$, $140)=8.74, p=.004, \eta_{\mathrm{p}}{ }^{2}=.06$; a main effect of Avoid Instruction, $F(1,140)=7.22, p=.008, \eta_{\mathrm{p}}{ }^{2}=.05$, and a significant Example Exposure $X$ Avoid Instruction interaction, $F(1,140)=6.52, p=.012, \eta_{\mathrm{p}}{ }^{2}=.04$. The NoExamples+NoAvoid group rated the familiarity of their generated ideas as significantly higher than the Examples+ Avoid group, $t(70)=4.07, p<.001, d=0.96$, the Examples+NoAvoid group, $t(70)=4.16, p<.001, d=0.98$, and the NoExamples+Avoid group, $t(70)=3.79, p<.001$, $d=0.89$, which did not differ from each other. For the planned comparison, the Examples+Avoid group rated the familiarity of their ideas $(M=2.92, S D=1.32)$ marginally less than that of all other conditions $(M=3.34, S D=1.31), t(142)=1.69, p$ $=.094, d=0.33$. For the questions asked only of the examples groups, there were no significant effects.

Example recall For example recall, only 14 observations were available for the Examples+Avoid group, and the comparison of means revealed no significant difference, $t(48)=0.06$, $p=.954, d=0.04$.

\section{Discussion}

The first experiment investigated whether instructing people to avoid a list of common example ideas during idea 
Table 2 Mean ratings for the 12 post-AUT questionnaire items across all experiments and conditions

\begin{tabular}{|c|c|c|c|c|}
\hline & \multicolumn{4}{|c|}{ Experiment 1 (Verbal Examples) } \\
\hline & $\begin{array}{l}\text { NoExamples+ } \\
\text { NoAvoid }\end{array}$ & $\begin{array}{l}\text { Examples+ } \\
\text { NoAvoid }\end{array}$ & $\begin{array}{l}\text { NoExamples+ } \\
\text { Avoid }\end{array}$ & $\begin{array}{c}\text { Examples+ } \\
\text { Avoid }\end{array}$ \\
\hline Difficult & 3.11 & 3.25 & 3.28 & 3.36 \\
\hline StuckGeneral & 3.28 & 3.72 & 3.67 & 3.58 \\
\hline StuckOnObvious & 3.19 & 3.25 & 2.89 & 3.11 \\
\hline EarlierIdeasGotInWay & 3.33 & 3.25 & 3.06 & 3.19 \\
\hline CreativityAboveAverage & 3.00 & 3.00 & 2.75 & 2.86 \\
\hline BrandNewIdeas & 2.75 & 3.19 & 3.22 & 3.25 \\
\hline FamiliarIdeas & 4.08 & 2.94 & 3.00 & 2.92 \\
\hline FiveIdeasGotInWay & - & 4.14 & - & 3.78 \\
\hline FiveIdeasDistracting & - & 3.17 & - & 3.17 \\
\hline FiveIdeasHelpful & - & 2.92 & - & 2.92 \\
\hline FiveIdeasHelpedAvoid & - & 3.50 & - & 3.61 \\
\hline \multirow[t]{3}{*}{ BuiltOffFiveIdeas } & - & 2.97 & - & 2.61 \\
\hline & \multicolumn{4}{|c|}{ Experiment 2 (Visual Examples) } \\
\hline & $\begin{array}{l}\text { NoExamples+ } \\
\text { NoAvoid }\end{array}$ & $\begin{array}{l}\text { Examples+ } \\
\text { NoAvoid }\end{array}$ & $\begin{array}{l}\text { NoExamples+ } \\
\text { Avoid }\end{array}$ & $\begin{array}{c}\text { Examples+ } \\
\text { Avoid }\end{array}$ \\
\hline Difficult & 3.31 & 3.31 & 3.58 & 3.56 \\
\hline StuckGeneral & 3.44 & 3.61 & 4.17 & 3.78 \\
\hline StuckOnObvious & 3.17 & 3.53 & 3.75 & 3.39 \\
\hline EarlierIdeasGotInWay & 3.36 & 2.75 & 3.50 & 3.11 \\
\hline CreativityAboveAverage & 2.81 & 2.53 & 2.61 & 2.69 \\
\hline BrandNewIdeas & 2.83 & 2.64 & 2.78 & 3.22 \\
\hline FamiliarIdeas & 3.00 & 3.28 & 3.53 & 2.78 \\
\hline FiveIdeasGotInWay & - & 4.14 & - & 3.97 \\
\hline FiveIdeasDistracting & - & 3.78 & - & 3.06 \\
\hline FiveIdeasHelpful & - & 2.81 & - & 3.00 \\
\hline FiveIdeasHelpedAvoid & - & 3.36 & - & 3.92 \\
\hline BuiltOffFiveIdeas & - & 3.22 & - & 2.92 \\
\hline
\end{tabular}

Note. The last five questions were only asked of the examples groups

generation would facilitate the production of original ideas, or if it would lead to mental fixation on example ideas. The results demonstrated that this condition improved originality on the AUT compared to all other conditions. This supports the notion that having a sense of which ideas are unoriginal (direction) at the outset of idea production can be beneficial in shifting people's attention toward more novel approaches. This condition may promote originality by making people reflect more on their idea generation rather than retrieving familiar ideas or ideas based closely on familiar ideas. This may be evidenced by the tendency to see longer idea response times in the examples conditions.

This pattern of results also rules out two alternative explanations. First, this benefit did not result from mere exposure to example ideas. There was no improvement in originality in the condition in which people viewed common example ideas without any additional instruction regarding those examples (Examples+NoAvoid), meaning exposure to the examples themselves did not stimulate creativity.
Second, this benefit was not due to the more general instruction to avoid ideas that may be common. This is evidenced by the fact that the originality of the NoExamples+Avoid group was not any higher than that of the NoExamples+ NoAvoid group. Thus, the benefit was not merely due to extra "encouragement" to avoid obvious ideas. Rather, there is something about the combination of the avoid instructions and the list of specific common examples that is beneficial. Additionally, when examining the proportion of original ideas (i.e., ratio scores that adjusted for fluency) rather than summative originality scores, the pattern remained the same indicating the effects were not a result of differences in fluency. The analysis of the time stamp of example responses also rules out the possibility that the no-examples groups simply spent all their time outputting example responses, leaving them with little room to think of more original ideas. The time stamp of the last example response indicates that sufficient time remained for these conditions to potentially generate originally ideas. 
Despite the differences in creative performance, there was little difference in average idea response times, time spent generating example ideas, participants' post-AUT questionnaire ratings for perceptions of difficulty, feelings of being stuck, or overall creativity. However, when further probed about the degree to which they thought of familiar ideas, the ratings seemed to differentiate by condition. The NoExamples+NoAvoid condition indicated that they generated many familiar ideas. This indicates that upon further prompting, the groups showed some awareness of the unoriginal quality of their ideas.

The results of Experiment 1 also replicate prior serial position effects in the AUT. In general, later ideas were more likely to be original. In the NoExamples+NoAvoid condition, this is not surprising because it is expected that people start off with the most accessible (and unoriginal) ideas, and only later do they reach more novel ones. Interestingly, however, this improvement over time was also seen in the Examples+Avoid condition - people not only began with original ideas but tended to increase their likelihood of originality over time. This may suggest that the combined manipulation condition was not only effective in helping them avoid the most obvious ideas, but it also helped them approach the task in an overall more productive manner.

Taken together, the results of Experiment 1 run counter to the fixation account and suggest that exposure to common example ideas can be helpful when they are framed as unoriginal ideas to try to avoid. This provides support for the direction account.

\section{Experiment 2}

In Experiment 1, when a set of common ideas was presented along with instructions highlighting the unoriginality of these common ideas and encouraging people to avoid them, a benefit was seen in originality. However, the results of Experiment 1 also suggested that mere exposure to examples without instructions to avoid them did not produce fixation in the sense of reduced originality relative to a group that received no examples and no avoid instructions. A wide set of findings on fixation in creative problem solving suggests that a fixation effect should result from exposure to common examples. For example, robust fixation effects occur in the RAT when misleading associates of RAT items are activated just prior to or during problem solving attempts (Koppel \& Storm, 2014; Smith \& Blankenship, 1991; Wiley, 1998). People who do not receive this exposure are more likely to solve the RAT problems than people who do. Both the RAT and AUT involve going beyond obvious responses in favor of more remote response. However, fixation effects in the RAT and the AUT may not be directly comparable. The problem space of the RAT is restricted to the verbal domain, particularly at the lexico-semantic level. What makes experimental inductions of fixation so powerful in the RAT is that success in this task involves retrieving specific lexicosemantic associations. When this lexico-semantic space is occupied by the activation of misleading associates, it can be very difficult to "un-see" these incorrect solutions. In contrast, in AUT there is no single correct solution. Although verbally mediated, generating ideas involves imagining objects being manipulated - in ways that break from their normal functions. Although the example ideas provided in Experiment 1 were common ones, their verbal mode of presentation may not have strongly interfered with the processes involved in imagining new uses. There is good theoretical and empirical reason to think the AUT involves visual mental imagery. Cognitive and neural processes involved in detailed episodic simulations seem to be important for AUT performance (Madore et al., 2015, 2016; Mullally \& Maguire, 2014), and thinking of uncommon uses involves patterns of brain activation that involve mental imagery (Benedek et al., 2018; Benedek, et al., 2014; Chrysikou \& Thompson-Schill, 2011).

Other work also suggests that a visual depiction of the common example ideas may act as a stronger source of fixation. Work by Chrysikou et al. (2016) demonstrated that AUT prompts presented in visual rather than verbal form may make knowledge of the typical actions and functions of objects more salient. This resulted in ideas that were closely related to an object's typical function. Additionally, much of the prior work suggesting a negative impact of fixation on idea generation involved visual or pictorial depictions of examples (Cardoso \& Badke-Schaub, 2011; Chrysikou \& Weisberg, 2005; Jansson \& Smith, 1991; Maier, 1931; Marsh et al., 1996; Smith et al., 1993). Thus, the primary purpose of Experiment 2 was to test whether fixation (reduced originality) would be observed as a result of mere exposure to the five example ideas presented in visual form, and whether instructions to avoid these ideas would serve to alter that fixation.

The design of Experiment 2 was parallel to that of Experiment 1 , but the verbal examples were replaced with visually depicted examples. If the visual examples make the typical functions more salient, then mere exposure to these images (Examples+NoAvoid) should lead to reduced originality relative a group who had no exposure to examples and no avoid instructions (NoExamples+ NoAvoid). The images did not include a verbal label. This decision was motivated by Chrysikou et al. (2016), who found that images of objects with no label led to more top-down driven responses (i.e., more related to typical function) than a labels-only condition and an images-plus-labels condition. As in Experiment 1, there was also a group that viewed the common example ideas in picture form with the addition of the avoid instructions (Examples+ Avoid). If the Examples+NoAvoid group experiences fixation from the examples, the question remains as to whether this fixation can be countered by the avoid instructions. Chrysikou and Weisberg (2005) found that "de-fixating" instructions helped participants to avoid using flawed examples of designs. This would suggest that the Examples+Avoid condition would produce as many original ideas as or more than the NoExamples+NoAvoid 
group. On the other hand, if the visual examples produce a very strong fixation effect, then the avoid instructions may simply be ineffective in overcoming this fixation, and this would result in less originality than the NoExamples+NoAvoid condition. To make the design parallel to that of Experiment 1, a NoExamples+ Avoid condition was also included in which participants did not view example ideas but were instructed to generally avoid thinking of common ideas that many others might think of. As in Experiment 1, a planned comparison between the Examples+ Avoid condition to all others was conducted.

\section{Method}

Participants and design Participants were 144 undergraduates (108 female, $M_{\text {age }}=19.01$ years, $S D=1.26$ ) recruited from the University of Illinois at Chicago psychology subject pool who were compensated with course credit. As in Experiment 1, the design was a $2 \times 2$ between-subjects design crossing Example Exposure (examples vs. no examples) and Instruction (avoid vs. no avoid) with 36 participants randomly assigned to each condition.

Materials The materials were the same as for the first experiment, except instead of a verbal label for each example, a fullcolor photographic image of each example was selected from an internet search (see Fig. 3). These images were cropped to $200 \times 200$ pixel squares.

Procedure All procedures, including instructions and coding, were the same as in Experiment 1, except that the verbal example ideas were replaced with the images in roughly the same location on the screen.

\section{Results}

Number of original responses (originality) ${ }^{4}$ There was no significant main effect of Example Exposure on summative originality scores, $F(1,140)=0.96, p=.330, \eta_{\mathrm{p}}{ }^{2}=$ .01 , and no significant main effect of Avoid Instruction, $F(1,140)=2.15, p=.145, \eta_{\mathrm{p}}{ }^{2}=.02$. However, there was a significant interaction, $F(1,140)=4.21, p=.042, \eta_{\mathrm{p}}{ }^{2}=$ .03. As shown in Fig. 4, the Examples+NoAvoid group generated fewer original ideas than the NoExamples+ NoAvoid group, $t(70)=2.08, p=.018, d=0.57$, as well

\footnotetext{
${ }^{4}$ As in Experiment 1, parallel analyses using ratio scores yielded results similar to the primary analyses. There was no main effect of Example Exposure $F(1,140)=0.39, p=.536, \eta_{\mathrm{p}}{ }^{2}=.003$, no main effect of Avoid Instruction, $F(1$, $140)=1.78, p=.184, \eta_{\mathrm{p}}{ }^{2}=.01$, and a significant interaction, $F(1,140)=6.05$, $p=.015, \eta_{\mathrm{p}}{ }^{2}=.04$. For the planned comparison, the Examples+Avoid group had a marginally higher originality proportion $(M=.59, S D=.32)$ than the other conditions $(M=.47, S D=.35), t(142)=1.81, p=.072, d=0.35$. The Examples+NoAvoid group had a lower originality proportion $(M=.38, S D=$ $.34)$ than the NoExamples+NoAvoid group $(M=.55, S D=.35), t(70)=2.16, p$ $=.034, d=0.51$.
}

as the Examples+Avoid group, $t(70)=2.98, p=.004, d=$ 0.70. Unlike in Experiment 1, the originality of the Examples+Avoid group did not significantly surpass that of all other groups, $t(142)=1.55, p=.149, d=0.29$.

Originality across idea position As shown in Fig. 5, an increase in the probability of original ideas across idea position occurred in the NoExamples+NoAvoid condition $(\beta=0.17, S E=0.06, Z$ $=2.87, p=.004)$, the NoExamples+Avoid condition $(\beta=0.15$, $S E=0.06, Z=2.54, p=.011)$. However, the effect of idea position was not significant in the Examples+NoAvoid condition ( $\beta=0.11, S E=0.10, Z=1.10, p=.272)$, nor in the Examples+ Avoid condition $(\beta=0.05, S E=0.07, Z=0.71, p=.476)$.

Other AUT measures The means for the other dependent measures are displayed in Table 3.

Fluency For total fluency (summative), there was a marginal main effect of Example Exposure with a trend toward higher total fluency in the no-examples $(M=5.31, S D=2.82)$ than examples groups $(M=4.47, S D=2.32), F(1,140)=3.71, p=$ $.056, \eta_{\mathrm{p}}{ }^{2}=.03$. There was no effect of Avoid Instruction, no interaction, $F(1,140)=0.004, p=.95, \eta_{\mathrm{p}}{ }^{2}=.00$, and no difference in the planned comparison between Examples+Avoid and the other conditions, $t(142)=0.96, p=.339, d=0.19$.

For the number of example responses (summative), ${ }^{5}$ there was a main effect of Example Exposure with more example ideas generated in the no-examples groups $(M$ $=1.79, S D=1.29)$ than examples groups $(M=0.94$, $S D=1.34), F(1,140)=14.94, p<.001, \eta_{\mathrm{p}}{ }^{2}=.10$. There was no effect of Avoid Instruction, $F(1,140)=$ $1.16, p=.283, \eta_{\mathrm{p}}{ }^{2}=.01$, and no interaction $F(1,140)$ $=0.90, p=.343, \eta_{\mathrm{p}}{ }^{2}=.01$. For the planned comparison, the Examples+Avoid generated fewer example responses $(M=0.72, S D=1.26)$ than the other conditions $(M=1.58, S D=1.35), t(142)=3.36, p=.001, d$ $=0.69$.

For the number of non-example responses (summative), there was no significant effect of Example Exposure, $F(1$, $140)=0.02, p=.903, \eta_{\mathrm{p}}^{2}=.00$, Avoid Instruction, $F(1$, $140)=0.47, p=.492, \eta_{\mathrm{p}}{ }^{2}=.00$, no interaction $F(1,140)=$ $1.20, p=.276, \eta_{\mathrm{p}}{ }^{2}=.01$ and no difference in the planned comparison, $t(142)=1.11, p=.271, d=0.22$.

\footnotetext{
${ }^{5}$ For the ratio of example responses, there was a main effect of Example Exposure, $F(1,140)=19.31, p<.001, \eta_{\mathrm{p}}{ }^{2}=.12$, with a greater proportion of example responses (and lower proportion of non-example response) in the No Examples condition $(M=.33, S D=.24)$ than in the Examples condition $(M$ $=.17, S D=.22$ ), no main effect of Avoid Instruction, $F(1,140)=2.47, p=$ $.118, \eta_{\mathrm{p}}{ }^{2}=.02$, and no interaction, $F(1,140)=1.46, p=.230, \eta_{\mathrm{p}}{ }^{2}=.01$. For the planned comparison, the Examples+Avoid group generated a smaller proportion of example responses (and greater proportion of non-example responses) $(M=.12, S D=.19)$ than the other conditions $(M=.29, S D=.23), t(142)=$ $4.08, p<.001, d=0.83$.
} 

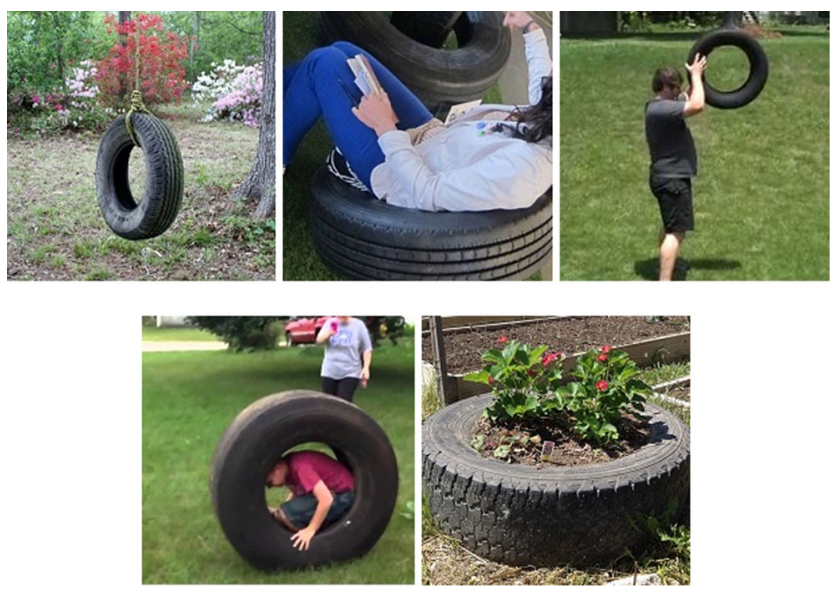

Fig. 3 Visual examples displayed in Experiment 2

Flexibility $^{6}$ For flexibility (summative number of categories computed excluding examples), there was no effect of Example Exposure, $F(1,140)=0.57, p=.450, \eta_{\mathrm{p}}{ }^{2}=.004$, no effect of Avoid Instruction, $F(1,140)=0.40, p=.529, \eta_{\mathrm{p}}{ }^{2}$ $=.003$, and no interaction, $F(1,140)=0.78, p=.378, \eta_{\mathrm{p}}{ }^{2}=$ .01 . There was no difference in the planned comparison, $t(142)=1.32, p=.189, d=0.24$.

Average response time per idea For idea response time, there was a marginal main effect of Example Exposure, with a trend toward longer response times in the examples groups $(M=$ $51.2 \mathrm{~s}, S D=33.3 \mathrm{~s})$ than the no-examples groups $(M=41.5 \mathrm{~s}$, $S D=27.0 \mathrm{~s}), F(1,140)=3.68, p=.057, \eta_{\mathrm{p}}{ }^{2}=.03$. There was no effect of Avoid Instruction $F(1,140)=0.002, p=.967, \eta_{\mathrm{p}}{ }^{2}$ $=.00$, no interaction, $F(1,140)=0.04, p=.846, \eta_{\mathrm{p}}{ }^{2}=.00$ and no difference in the planned comparison, $t(142)=1.02, p=$ $.311, d=0.20$.

Once again, in order to provide some assessment of how long participants may have spent generating the example ideas (particularly those in the no-examples conditions), the second timing measure that was calculated was the time stamp following the last example idea that was generated. There was no main effect of Example Exposure, $F(1,90)=0.37, p=.543$, $\eta_{\mathrm{p}}{ }^{2}=.00$, no main effect of Avoid Instruction, $F(1,90)=0.14$, $p=.748, \eta_{\mathrm{p}}{ }^{2}=.00$, and no interaction, $F(1,90)=0.15, p=$ $.700, \eta_{\mathrm{p}}{ }^{2}=.00$. For the planned comparison of Examples+ Avoid and all other conditions, there was no significant difference, $t(92)=0.66, p=.513, d=0.20$.

Post-AUT questionnaire The means for the responses to the post-task questionnaire are shown in Table 2. For questions asked of all conditions, differences were observed on three of the seven questions. For the amount of familiar ideas

\footnotetext{
${ }^{6}$ For the flexibility ratio score, there was no main effect of Example Exposure, $F(1,140)=0.21, p=.651, \eta_{\mathrm{p}}{ }^{2}=.001$, Avoid Instruction, $F(1,140)=0.10, p=$ $.755, \eta_{\mathrm{p}}{ }^{2}=.001$, nor an interaction, $F(1,140)=1.81, p=.181, \eta_{\mathrm{p}}{ }^{2}=.01$, nor a difference in the planned comparison, $t(142)=1,23, p=.223, d=0.24$.
}

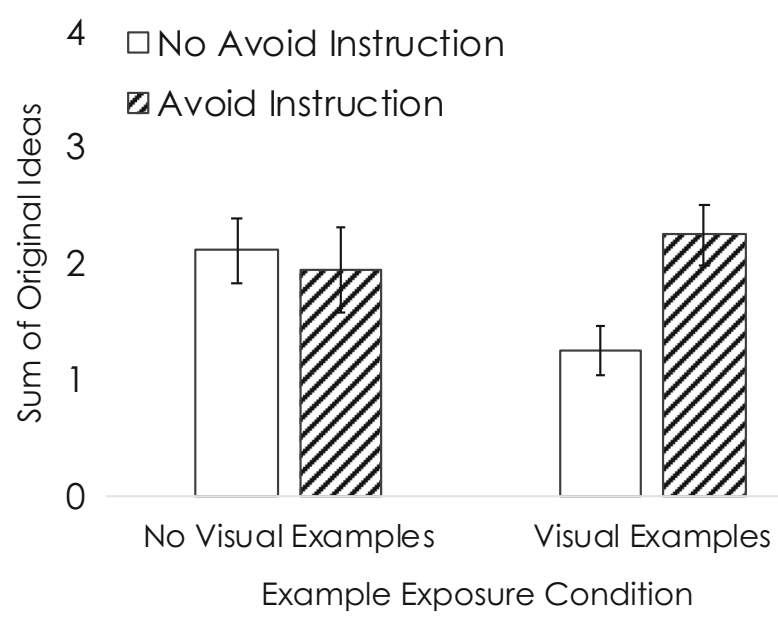

Fig. 4 Mean number of original ideas by condition in Experiment 2. Error bars represent standard errors (1 SEM)

generated there was no main effect of Example Exposure, $F(1,140)=1.63, p=.204, \eta_{\mathrm{p}}{ }^{2}=.01$, or Avoid Instruction, $F(1,140)=0.01, p=.940, \eta_{\mathrm{p}}{ }^{2}=.00$. There was an Example Exposure $\mathrm{x}$ Avoid Instruction interaction, $F(1,140)=7.72, p$ $=.006, \eta_{\mathrm{p}}{ }^{2}=.05$. The Examples+Avoid group reported generating fewer familiar ideas than the NoExamples+Avoid group, $t(70)=3.03, p=.003, d=0.64$, and marginally fewer familiar ideas than the Examples+NoAvoid group, $t(70)=$ $1.84, p=.071, d=0.43$. For the planned comparison, the Examples+Avoid group rated familiarity higher than all the other groups, $t(142)=2.28, p=.024, d=0.43$.

For the degree to which earlier ideas were rated as getting in the way of later ideas, there was a main effect of Example Exposure, $F(1,140)=6.13, p=.014, \eta_{\mathrm{p}}{ }^{2}=.04$, with the no examples groups reporting that earlier ideas got in the way $(M$ $=3.43, S D=1.12)$ more than the examples groups $(M=2.93$, $S D=1.29)$. There was no effect of Avoid Instructions, $F(1$, $140=1.53, \mathrm{p}=.218, \eta_{\mathrm{p}}{ }^{2}=.01$, no interaction, $F(1,140=0.30$, $\mathrm{p}=.583, \eta_{\mathrm{p}}{ }^{2}=.002$, and no difference in the planned comparison, $t(142)=0.39, p=.698, d=0.07$.

For the rating of feeling stuck while generating ideas, there was a main effect of Avoid Instruction, $F(1,140)=5.90, p=.016, \eta_{\mathrm{p}}{ }^{2}$ $=.04$, with groups receiving avoid instructions reporting being more stuck $(M=3.97, S D=1.05)$ than groups without avoid instructions $(M=3.53, S D=1.15)$. There was no effect of Example Exposure, $F(1,140)=0.37, p=.545, \eta_{\mathrm{p}}{ }^{2}=.003$, no interaction, $F(1,140)=2.31, p=.131, \eta_{\mathrm{p}}{ }^{2}=.02$, and no difference in the planned comparison, $t(142)=0.13, p=.901, d=0.03$.

For the five questions asked only of the two examples groups, the Examples+NoAvoid group reported that the five examples were more distracting than the Examples+Avoid group, $t(70)=2.14, p=.036, d=0.50$. Additionally, the Examples+Avoid group tended to report that the five example ideas helped them avoid obvious ideas more than the Examples+NoAvoid group, although this difference was marginal, $t(62)=1.91, p=.061, d=0.45$. 
No Examples + No Avoid

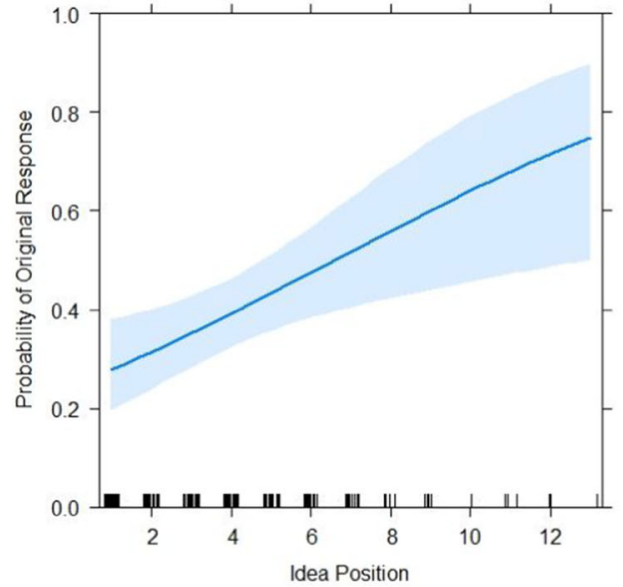

Examples + No Avoid

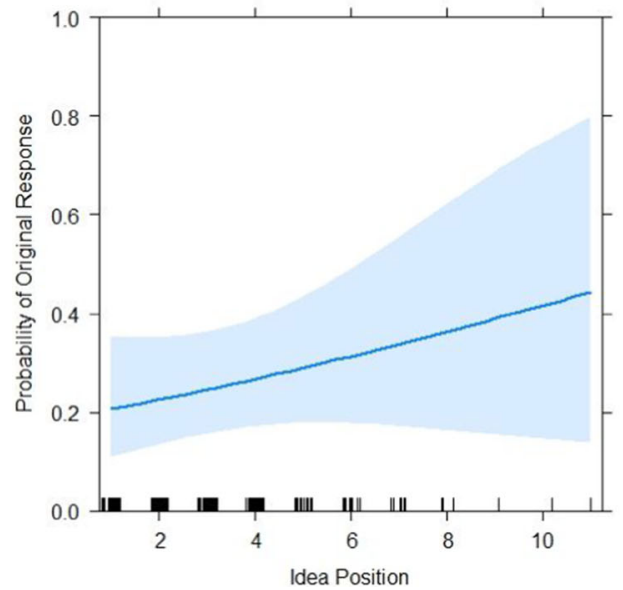

No Examples + Avoid

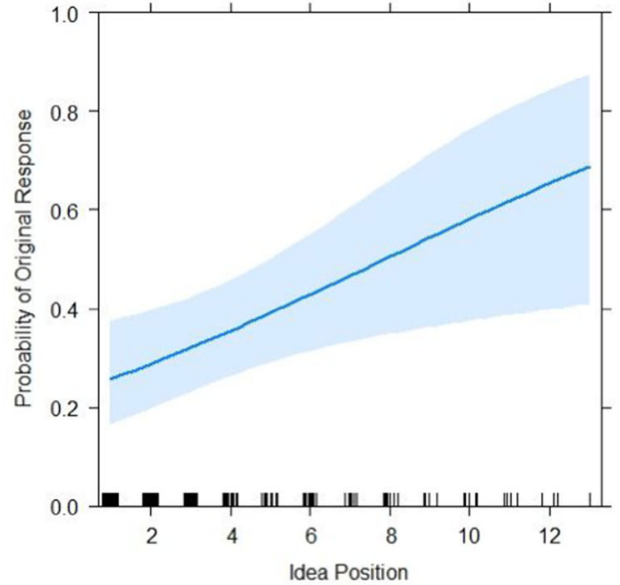

Examples + Avoid

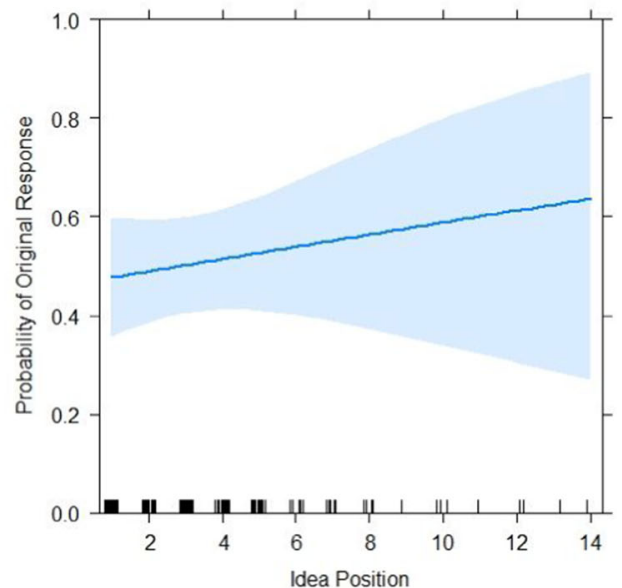

Fig. 5 Effects of idea position on likelihood of original responses across warning and visual example conditions in Experiment 2. Shaded areas indicate $95 \%$ confidence bands

In summary, participants exposed to visual examples reported that their earlier ideas interfered with later ideas less than participants not exposed to visual examples. The avoid instructions generally led to a greater feeling of being stuck.
The group given examples and no avoid instruction also reported feeling more distracted by the examples. Additionally, consistent with Experiment 1, the instruction to avoid examples led to a reduction in rated familiarity of ideas.

Table 3 Mean values for AUT measures by condition in Experiment 2

\begin{tabular}{lcccc}
\hline & \multicolumn{1}{l}{$\begin{array}{l}\text { NoExamples+NoAvoid } \\
M(S D)\end{array}$} & $\begin{array}{l}\text { Examples+NoAvoid } \\
M(S D)\end{array}$ & $\begin{array}{l}\text { NoExamples+Avoid } \\
M(S D)\end{array}$ & $\begin{array}{l}\text { Examples+ Avoid } \\
M(S D)\end{array}$ \\
\hline Fluency (Total) & $5.39(2.61)$ & $4.42(2.13)$ & $5.22(3.04)$ & $4.53(2.52)$ \\
Fluency (Example responses) & $1.81(1.21)$ & $1.17(1.40)$ & $1.78(1.38)$ & $0.72(1.26)$ \\
Fluency (Non-example responses) & $3.53(2.12)$ & $3.19(1.55)$ & $3.39(2.44)$ & $3.81(2.03)$ \\
Flexibility (Non-example responses) & $2.69(1.21)$ & $2.67(1.17)$ & $2.64(1.25)$ & $3.00(1.60)$ \\
Average Response Time per Idea (s) & $40.85(24.86)$ & $51.60(37.23)$ & $42.05(29.41)$ & $50.82(29.39)$ \\
Last Example Response Time Stamp (s) & $110.09(54.54)$ & $107.36(55.73)$ & $110.89(60.02)$ & $98.60(55.20)$ \\
Example Recall (Proportion) & - & $.91(.13)$ & - & $.81(.18)$ \\
\hline
\end{tabular}


Example recall For example recall, the Examples+NoAvoid group recalled significantly more of the example ideas than the Examples+Avoid group, $t(70)=2.56, p<.013, d=0.64$. Additionally, as shown in Table 3, the high level of recall indicates that participants were able to recognize the uses depicted in the example photographs. This means the lack of verbal labels for the images did not affect participants' ability to identify the uses.

\section{Discussion}

In contrast to the results of Experiment 1, visual displays of the example ideas led to a different pattern of originality. The most critical finding was that being shown visual examples of ideas - without specific instruction to avoid them - led to reduced originality relative to the NoExamples+NoAvoid condition. This is consistent with the notion of mental fixation arising from exposure to example ideas which produces diminished originality. Additional pieces of evidence for this come from the Examples+NoAvoid group reporting that the five examples were more distracting than the Examples+ Avoid condition, as well as showing a higher level of recall of the example ideas. The lack of fixating effects in Experiment 1 may be due to the fact that the examples were presented in verbal form, which possibly did little to interfere with creative idea generation in the AUT. However, when common example ideas are displayed in picture form, this may make typical object functions much more salient, leading to a more biased mental set during idea generation. This result extends the findings of Chrysikou et al. (2016), who found that visual depictions of just the object as part of the AUT prompt made typical uses more salient. One possibility is that participants were not as able to recognize the example uses depicted in the images as well as in Experiment 1; however, the high level of recall of the example indicates that participants were able to accurately identify the uses depicted in the images. Additionally, as in Experiment 1, when examining the proportion of original ideas rather than summative originality scores, the pattern remained the same indicating the effects were not a result of differences in fluency.

Further evidence for fixation comes from the serial position analysis. While the no-examples conditions showed a greater likelihood of originality in later responses, the conditions that viewed visual examples did not significantly improve across idea position, as is otherwise common in idea generation tasks.

Interestingly, the avoid instructions (Examples+Avoid) helped to counteract fixating effects in the sense that originality in this condition was significantly better than that of the Examples+NoAvoid group. However, this group did not reach originality levels that were significantly better than the NoExamples+NoAvoid condition. Thus, these results indicate that while avoid instructions may help people gain an advantage in originality when those example ideas are verbally presented (Experiment 1), they may not be as helpful when they are visually presented (Experiment 2).

\section{General discussion}

Generating creative ideas requires shifting attention away from familiar approaches and easily accessible ideas. When given creative prompts, such as the AUT, we are not likely to just simply "draw a blank," but rather an initial set of familiar ideas may arise and these ideas can easily occupy our attention. This can be detrimental when the goal is to be different and break away from common approaches in favor of novel ones - people may easily be led down the wrong path by retrieving familiar ideas (Beaty \& Silvia 2012; Gilhooly et al., 2007).

These experiments explored whether providing people with examples of common ideas that are specifically framed as unoriginal - that is, encouraging people to think of ideas that are different from common ideas - could have a positive impact on originality in the AUT. A large body of literature suggests that mental fixation in creative contexts can arise from exposure to misleading information (Jansson \& Smith 1991; Kohn \& Smith, 2011; Koppel \& Storm, 2014; Marsh et al., 1996; Smith \& Blankenship, 1991; Smith et al., 1993; Storm \& Hickman, 2015; Wiley, 1998). On the other hand, it could be important to have a sense of the ideas that are unoriginal at the outset of idea generation - in other words, it is good to know what has been done so that one might direct their attention away from these solutions. The tension between these two ideas is reflected in the results across these two experiments - example exposure had very different effects on originality depending on instructions and the presentation modality of the examples.

In Experiment 1, when the common ideas were presented verbally, being instructed to avoid the common ideas improved originality relative to all other conditions. This suggests that the common examples can serve as a useful reference point for generating more novel ideas. Additionally, the benefit of this manipulation was not due to mere example exposure. When the example ideas were presented without instructions to avoid, there was no improvement in originality. Moreover, the effect was not simply due to additional general encouragement, as the groups that received a general instruction to avoid common ideas without reference to any specific examples did not outperform the baseline groups.

One concern could be that the no-examples groups simply spent too much time outputting the example responses and therefore had little opportunity to generate original ideas before the time ran out. However, there are a few arguments against this interpretation. First, as shown in Tables 1 and 2, participants typically provided around five ideas in the 3-min time limit, suggesting people generally did not maximize their time allotment (approximately $40 \mathrm{~s}$ were spent per idea) 
Second, the analysis of the time stamp of the participants' last example response also argues against this interpretation. For the no-examples participants, the typical time stamp of their last example response was about $100 \mathrm{~s}$ into the 3-min ideageneration period. In other words, they had plenty of time left to think of other potentially original ideas. Additionally, the results of the analysis of ratio scores (non-example originality/ non-example fluency) followed the same pattern as the summative originality scores, indicating that the no-examples groups' scores were not simply lowered due to their more frequent generation of example (i.e., unoriginal) ideas. A closely related argument can be made from the finding that the Examples+NoAvoid condition also generated significantly fewer example responses than the no-examples conditions. Despite this group also outputting fewer example responses (unoriginal responses), their originality was not boosted. If the benefit of the Examples+Avoid condition were purely due to the no-examples groups running out of time, then the Examples+NoAvoid condition should also have benefited from not spending as much time on these common examples. In other words, generating fewer example responses did not necessarily result in more originality.

With regard to the finding that simply instructing people to avoid ideas that others might think of (NoExamples+Avoid) did not improve originality, this suggests that such provision of a "general avoid" strategy is not helpful. However, this is not to say that other strategies could not be beneficial. Research has found that provision of other more specific strategies can improve the originality of ideas (Forthmann et al., 2019; Nusbaum \& Silvia, 2011; Wilken et al., 2019), such as imagining the disassembly or component parts of an object.

Although one may expect that avoid instructions could paradoxically lead to further activation of the common examples (Wegner, 1994; Wiley, 1998), the finding that instructions to avoid the common examples improved originality in Experiment 1 is consistent with suggestions that people are effective at inhibiting explicitly activated representations (Angello, Storm, \& Smith, 2015). These results can also be interpreted from the perspective of Jacoby's (1991) distinction between automatic and controlled memory processes, in that fixation effects may be resulting from automatic activation of common ideas in memory, while instructions to avoid common ideas may be encouraging relatively greater use of controlled or strategic searches of memory. This would be consistent with other work that has argued that strategic processing can benefit creative idea generation (Benedek et al., 2018). Although the present studies were not designed to test for this possibility, future work may seek to explore this mechanism.

Experiment 2 demonstrated that examples can have a fixating effect specifically when the examples were presented in visual rather than verbal form. With visual examples and no specific instruction to think of different ideas, this led to an impairment in originality. Even when instructed to avoid the visual examples and think of different ideas, this failed to produce gains in originality as it did in Experiment 1. In this way, the current results are similar to those of Chrysikou and Weisberg (2005) who found that visually depicted example designs caused people to conform to the examples, that de-fixation instructions mitigated this conformity, but did not make them produce designs of greater dissimilarity than a control group.

The serial position analyses across experiments also shed further light on the differences that were seen across experiments due to the modality of the examples. As in prior research, an increase in originality was observed in all conditions in Experiment 1, which is common with typical divergent thinking instructions (Beaty \& Silvia, 2012; Benedek \& Neubauer, 2013; Christensen et al., 1957; George \& Wiley, 2019; Glaveanu et al., 2018; Hass, 2017; Hass \& Beaty, 2018; Kraus et al., 2019; Kudrowitz \& Dippo, 2013). Interestingly, this improvement in originality was also observed in the Examples+Avoid condition of Experiment 1 - people produced original ideas early on and increased their originality over time. In contrast, when presented with visual examples in Experiment 2, this serial position effect was attenuated - people did not significantly increase their likelihood of originality in later ideas, which provides a further indication of fixation from visual examples.

Thus, visual examples appear to be a powerful fixating force when generating novel object uses. Part of the reason for these different effects across experiments may be that the AUT involves some degree of visual imagination of objects being manipulated (Benedek et al., 2018; Benedek et al., 2014, Chrysikou et al., 2016, Chrysikou \& Thompson-Schill, 2011; Madore et al., 2015, 2016). When presented with photos of common uses, this may further increase the salience of obvious object manipulations and impair creative thinking. However, when these common uses are described in simple verbal statements, this interference may not be present. Further, when provided with the additional instructions encouraging avoiding the example ideas, this may help people separate these ideas as unoriginal, and shift their focus to different approaches that they would not readily consider. By having some sense at the outset of idea production of which specific uses are common people can discard those or similar ideas and think more carefully about their idea construction.

Although it has been suggested that visual object prompts may enhance activation of typical action information (Chrysikou et al., 2016) that could impair originality, future work should aim to clarify this mechanism while also addressing other potential ways that visual example exposure could have a negative impact. It is possible that the manipulation of verbal versus photographic example ideas may have varied multiple factors. The photographs in the present study may convey more (and more varied) information than the verbal description. For example, in the tire swing photograph, this may carry information not only about tire swings but about 
fun activities, outdoor activities, etc. Another possibility is that more complex visual examples are more difficult to suppress or inhibit, unlike simpler verbal responses (Angello et al., 2015).

There are several limitations to this line of experiments that should be noted. First, the hypotheses were tested using a particular creative problem-solving task (AUT) and only one object. It is possible that the benefits observed here are task or object specific, and these manipulations may benefit creative thinking only in these kinds of tasks, or for a specific subset of objects. There are many familiar, salient uses for common objects and being more aware of which ideas are unoriginal may be important. However, it is not clear if the results will generalize and whether this combination of manipulations would benefit performance on other sorts of creative problemsolving tasks. For example, some design tasks have demonstrated a constraining effect of examples, even when instructed to avoid those examples (Jansson \& Smith, 1991; Smith et al. 1993). Goldenberg and Wiley (2019) have found that brainstorming groups show more benefits from hearing ideas from others during collaboration on the AUT than on an "improvements" task. Additionally, Hass and Beaty (2018) recently demonstrated dissimilarities between the AUT and the consequences task, which prompts people to imagine consequences of novel scenarios (what if people no longer needed to sleep?). Performance may be more strongly influenced and fixated by prior knowledge in the AUT than on other idea generation tasks, including these improvements or consequences tasks.

A second set of limitations relates to the specifics of the procedure that was selected for these studies. For example, participants worked independently and data was collected as part of a laboratory study and it is unclear how these manipulations might affect idea generation in more collaborative contexts or outside the lab. Finally, participants had a very limited amount of time $(3 \mathrm{~min})$ in which to generate ideas. This choice was intentional, as there are likely multiple successful paths to creative idea generation (Baas, Roskes, Sligte, Nijstad, \& De Dreu, 2013), and more protracted periods of idea generation could have allowed for more equalization of originality across participants, which could have obscured effects. The main focus here was early stages of idea generation. While some recent work has found that performance on short and long time limits is highly correlated (Forthmann, Lips, Szardenings, Scharfen, \& Holling, 2018; Forthmann, Szardenings, \& Holling, 2018), future work should nonetheless address longer time scales and non-laboratory and/ or collaborative contexts.

Additionally, future research should examine how these effects may be moderated by individual differences. While the instructions to avoid common examples seemed to be generally beneficial, they may not be beneficial for all people. For example, research has demonstrated that individual differences related to executive control (e.g., general fluid intelligence) have a sizeable impact on creative problem-solving performance, and this relationship is further moderated by task conditions (Forthmann et al., 2019; Nusbaum \& Silvia, 2011; Nusbaum et al., 2014; Wilken et al., 2019). It is possible that avoiding common examples requires inhibitory control. When executive functioning is limited, this may be detrimental to performance by causing fixation on the examples (see Wiley, 1998, for ironic effects of warnings on fixation).

These results also have some potential practical implications. For example, when brainstorming ideas, it may be beneficial to keep a list of ideas that have been deemed noncreative available, so that teams can be sure to avoid such ideas. However, it is also important to consider the nature of examples. In the case of the AUT, visually presented ideas seem to be particularly likely to interfere with idea generation, which is ultimately counter-productive.

To conclude, these results point to a complex relationship between example exposure, modality, task instructions, fixation, and creative idea generation in the AUT. A major goal of creativity research is to understand the conditions that enhance or diminish novel idea generation. The results from the visually presented examples are more in line with prior work on mental fixation in creative problem solving because visual examples reduced originality. However, these experiments demonstrate that the effects of examples on creativity is not always negative. Encouraging people to avoid common examples is not necessarily detrimental and can even benefit originality in the AUT by providing direction to participants, so long as those ideas are presented in simple verbal form and with a warning.

Acknowledgements The authors were supported by a grant from the National Science Foundation (1535299) during the completion of this research at the University of Illinois of Chicago, and thank Amy Chou, Nida Fayyaz, Jessica McAleer, Shiwangi Pandya, and Fawn Wong for assistance in data collection and coding.

\section{Appendix 1}

\section{AUT instructions}

Examples+Avoid instructions:

Here are five of the most COMMON ideas people come up with for a car tire. These are less original ideas which many other people typically think of.

To help you come up with the most creative ideas as you can, this list will be available to help you avoid wasting time on these less original ideas. Since more creative ideas tend to be ones that many people would NOT think of, this list should help you generate the most original ideas as you can. See if 
you can avoid generating ideas like the ones in this list when you are thinking of your own new ideas."

Examples+No Avoid instructions:

Here are five example ideas. Please take a moment to review these examples. These examples will be on the screen during the task.

No Examples+Avoid instructions:

To help you come up with the most creative ideas as you can, try to avoid wasting time on less original ideas. Since more creative ideas tend to be ones that many people would NOT think of, this should help you generate the most original ideas as you can. See if you can avoid generating common ideas when you are thinking of your own new ideas."

\section{Appendix 2}

\section{Semantic categories of ideas}

\author{
air use \\ arts and crafts \\ boundary/barricade \\ buoyancy \\ clothes/fashion/jewelry \\ containing/storing/holding objects \\ décor \\ exercise/training/sports \\ fun/games/entertainment \\ furniture/house stuff \\ holding/weighing down \\ padding/protection/stabilization \\ vehicle-related \\ recycle/construction material \\ tool \\ weapons/harm/destruction
}

\section{References}

Agogué, M., Kazakçi, A., Hatchuel, A., Masson, P., Weil, B., Poirel, N., \& Cassotti, M. (2014). The impact of type of examples on originality: Explaining fixation and stimulation effects. The Journal of Creative Behavior, 48, 1-12.

Angello, G. A., Storm, B. C., \& Smith, S. M. (2015). Overcoming fixation with repeated memory suppression. Memory, 23, 381-389.

Baas, M., Roskes, M., Sligte, D., Nijstad, B. A., \& De Dreu, C. K. W. (2013). Personality and creativity: The dual pathway to creativity model and a research agenda. Social and Personality Psychology Compass, 7, 732-748.

Bates, D.M., Maechler, M., \& Bolker, B. (2012). lme4: Linear mixedeffects models using S4 classes. R package version 0.999999-0.
Beaty, R. E., Christensen, A. P., Benedek, M., Silvia, P. J., \& Schacter, D. L. (2017). Creative constraints: Brain activity and network dynamics underlying semantic interference during idea production. Neuroimage, 148, 189-196.

Beaty, R. E., \& Silvia, P. J. (2012). Why do ideas get more creative across time? An executive interpretation of the serial order effect in divergent thinking tasks. Psychology of Aesthetics, Creativity, and the Arts, 6, 309-319.

Benedek, M., Könen, T., \& Neubauer, A. C. (2012). Associative abilities underlying creativity. Psychology of Aesthetics, Creativity, and the Arts, 6, 273-281.

Benedek, M., \& Neubauer, A. C. (2013). Revisiting Mednick's model on creativity-related differences in associative hierarchies. Evidence for a common path to uncommon thought. The Journal of Creative Behavior, 47, 273-289.

Benedek, M., Schickel, R. J., Jauk, E., Fink, A., \& Neubauer, A. C. (2014). Alpha power increases in right parietal cortex reflects focused internal attention. Neuropsychologia, 56, 393-400.

Benedek, M., Schües, T., Beaty, R. E., Jauk, E., Koschutnig, K., Fink, A., \& Neubauer, A. C. (2018). To create or to recall original ideas: Brain processes associated with the imagination of novel object uses. Cortex, 99, 93-102.

Birch, H. G., \& Rabinowitz, H. S. (1951). The negative effect of previous experience on productive thinking. Journal of Experimental Psychology, 41, 121-125.

Camarda, A., Salvia, É., Vidal, J., Weil, B., Poirel, N., Houdé, O., ... Cassotti, M. (2018). Neural basis of functional fixedness during creative idea generation: An EEG study. Neuropsychologia, 118, 4-12.

Cardoso, C., \& Badke-Schaub, P. (2011). The influence of different pictorial representations during idea generation. The Journal of Creative Behavior, 45, 130-146.

Chen, C., Kasof, J., Himsel, A., Dmitrieva, J., Dong, Q., \& Xue, G. (2005). Effects of explicit instruction to "be creative" across domains and cultures. The Journal of Creative Behavior, 39, 89-110.

Christensen, P. R., Guilford, J. P., \& Wilson, R. C. (1957). Relations of creative responses to working time and instructions. Journal of Experimental Psychology, 53, 82-88.

Chrysikou, E. G., Motyka, K., Nigro, C., Yang, S., \& Thompson-Schill, S. (2016). Functional fixedness in creative thinking tasks depends on stimulus modality. Psychology of Aesthetics, Creativity, and the Arts, 10, 425-435.

Chrysikou, E. G., \& Thompson-Schill, S. (2011). Dissociable brain states linked to common and creative object use. Human Brain Mapping, $32,665-675$.

Chrysikou, E. G., \& Weisberg, R. W. (2005). Following the wrong footsteps: Fixation effects of pictorial examples in a design problemsolving task. Journal of Experimental Psychology: Learning, Memory, and Cognition, 31, 1134-1148.

Ezzat, H., Agogué, M., Le Masson, P., Weil, B., \& Cassotti, M. (2018). Specificity and abstraction of examples: Opposite effects on fixation for creative ideation. The Journal of Creative Behavior. Advance online publication. https://doi.org/10.1002/jocb.349

Ezzat, H., Camarda, A., Cassotti, M., Agogué, M., Houdé, O., Weil, B., \& Le Masson, P. (2017). How minimal executive feedback influences creative idea generation. PLoS ONE, 12, 1-10. https://doi.org/10. 1371/journal.pone. 0180458

Forthmann, B., Gerwig, A., Holling, H., Çelik, P., Storme, M., \& Lubart, T. (2016). The be-creative effect in divergent thinking: the interplay of instruction and object frequency. Intelligence, 57, 25-32.

Forthmann, B., Lips, C., Szardenings, C., Scharfen, J., \& Holling, H. (2018). Are speedy brains needed when divergent thinking is speeded-or unspeeded? The Journal of Creative Behavior. Advance online publication. https://doi.org/10.1002/jocb.350

Forthmann, B., Szardenings, C., \& Holling, H. (2018). Understanding the confounding effect of fluency in divergent thinking scores: 
Revisiting average scores to quantify artifactual correlation. Psychology of Aesthetics, Creativity, and the Arts. https://doi.org/ 10.1037/aca0000196

Forthmann, B., Wilken, A., Doebler, P., \& Holling, H. (2019). Strategy induction enhances creativity in figural divergent thinking. The Journal of Creative Behavior, 53, 18-29.

George, T. \& Wiley, J. (2019). Fixation, flexibility, and forgetting during alternate uses tasks. Psychology of Aesthetics, Creativity, and the Arts, 13, 305-313.

George, T., Wiley, J., Koppel, R. H., \& Storm, B. C. (2019). Constraining or constructive? The effects of examples on idea novelty. The Journal of Creative Behavior, 53, 396-403.

Gilhooly, K. J., Fioratou, E., Anthony, S. H., \& Wynn, V. (2007). Divergent thinking: Strategies and executive involvement in generating novel uses for familiar objects. British Journal of Psychology, 98, 611-625.

Glăveanu, V. P., Gillespie, A., \& Karwowski, M. (2018). Are people working together inclined towards practicality? A process analysis of creative ideation in individuals and dyads. Psychology of Aesthetics, Creativity, and the Arts, 13, 388-401

Goldenberg, O., Larson, J. R., Jr., \& Wiley, J. (2013). Goal instructions, response format, and idea generation in groups. Small Group Research, 44, 227-256.

Goldenberg, O., \& Wiley, J. (2019). Individual and group brainstorming: Does the question matter? Creativity Research Journal, 31, 261271.

Guilford, J. P. (1957). Creative abilities in the arts. Psychological Review, $64,110-118$.

Harrington, D. M. (1975). Effects of explicit instructions to "be creative" on the psychological meaning of divergent thinking test scores. Journal of Personality, 43, 434-454.

Hass, R. W. (2017). Tracking the dynamics of divergent thinking via semantic distance: Analytic methods and theoretical implications. Memory \& Cognition, 45, 233-244.

Hass, R. W., \& Beaty, R. E. (2018). Use or consequences: Probing the cognitive difference between two measures of divergent thinking. Frontiers in Psychology, 9, 1-15. https://doi.org/10.3389/fpsyg. 2018.02327

Jacoby, L. L. (1991). A process dissociation framework: Separating automatic from intentional uses of memory. Journal of Memory and Language, 30, 513-541.

Jansson, D. G., \& Smith, S. M. (1991). Design fixation. Design Studies, 12, 3-11.

Kohn, N. W., \& Smith, S. M. (2011). Collaborative fixation: Effects of others' ideas on brainstorming. Applied Cognitive Psychology, 25, 359-371.

Koppel, R. H., \& Storm, B. C. (2014). Escaping mental fixation: Incubation and inhibition in creative problem solving. Memory, $22,340-348$

Kraus, B., Cadle, C., \& Simon-Dack, S. (2019). EEG alpha activity is moderated by the serial order effect during divergent thinking. Biological Psychology, 145, 84-95.

Kudrowitz, B., \& Dippo, C. (2013). When does a paper clip become a sundial? Exploring the progression of originality in the alternative uses test. Journal of Integrated Design \& Process Science, 17, 3-18.

Madore, K. P., Addis, D. R., \& Schacter, D. L. (2015). Creativity and memory: Effects of an episodic-specificity induction on divergent thinking. Psychological Science, 26, 1461-1468.

Madore, K. P., Jing, H. G., \& Schacter, D. L. (2016). Divergent creative thinking in young and older adults: Extending the effects of an episodic specificity induction. Memory \& Cognition, 44, 974-988.

Maier, N. R. F. (1931). Reasoning in humans: II. The solution of a problem and its appearance in consciousness. Journal of Comparative Psychology, 12, 181-194.
Manske, M. E., \& Davis, G. A. (1968). Effects of simple instructional biases upon performance in the unusual uses test. The Journal of General Psychology, 79, 25-33.

Marsh, R. L., Landau, J. D., \& Hicks, J. L. (1996). How examples may (and may not) constrain creativity. Memory \& Cognition, 24, 669680.

Mednick, S. A. (1962). The associative basis of the creative process. Psychological Review, 69, 220-232.

Mullally, S. L., \& Maguire, E. A. (2014). Memory, imagination, and predicting the future: A common brain mechanism? The Neuroscientist, 20, 220-234.

Nusbaum, E. C., \& Silvia, P. J. (2011). Are intelligence and creativity really so different? Fluid intelligence, executive processes, and strategy use in divergent thinking. Intelligence, 39, 36-45.

Nusbaum, E. C., Silvia, P. J., \& Beaty, R. E. (2014). Ready, set, create: What instructing people to "be creative" reveals about the meaning and mechanisms of divergent thinking. Psychology of Aesthetics, Creativity, and the Arts, 8, 423-432.

Osborn, A. F. (1953). Applied imagination: Principles and procedures of creative thinking. New York, NY: Scribners.

Radel, R., Davranche, K., Fournier, M., \& Dietrich, A. (2015). The role of (dis)inhibition in creativity: Decreased inhibition improves idea generation. Cognition, 134, 110-120.

Rhodes, M. G., \& Castel, A. D. (2008). Metacognition and part-set cuing: Can interference be predicted at retrieval? Memory \& Cognition, 36, 1429-1438

Shin, H., Cotter, K. N., Christensen, A. P., \& Silvia, P. J. (2018). Creative fixation is no laughing matter: The effects of funny and unfunny examples on humor production. The Journal of Creative Behavior. Advance online publication. https://doi.org/10.1002/jocb.383

Sio, U. N., Kotovsky, K., \& Cagan, J. (2015). Fixation or inspiration? A meta-analytic review of the role of examples on design processes. Design Studies, 39, 70-99.

Smith, S. M., \& Blankenship, S. E. (1991). Incubation and the persistence of fixation in problem solving. The American Journal of Psychology, 104, 61-87.

Smith, S. M., Ward, T. B., \& Schumacher, J. S. (1993). Constraining effects of examples in a creative generation task. Memory \& Cognition, 21, 837-845.

Storm, B. C., \& Hickman, M. L. (2015). Mental fixation and metacognitive predictions of insight in creative problem solving. The Quarterly Journal of Experimental Psychology, 68, 802-813.

Storm, B. C., \& Patel, T. N. (2014). Forgetting as a consequence and enabler of creative thinking. Journal of Experimental Psychology: Learning, Memory, and Cognition, 40, 1594-1609.

Ward, T. B., Patterson, M. J., Sifonis, C. M., Dodds, R. A., \& Saunders, K. N. (2002). The role of graded category structure in imaginative thought. Memory \& Cognition, 30, 199-216.

Wegner, D. M. (1994). Ironic processes of mental control. Psychological Review, 101, 34-52.

Wiley, J. (1998). Expertise as mental set: The effects of domain knowledge in creative problem solving. Memory \& Cognition, 26, 716730 .

Wilken, A., Forthmann, B., \& Holling, H. (2019). Instructions moderate the relationship between creative performance in figural divergent thinking and reasoning capacity. The Journal of Creative Behavior. Advance online publication. https://doi.org/10.1002/jocb.392

Yagolkovskiy, S. R., \& Kharkhurin, A. V. (2016). The roles of rarity and organization of stimulus material in divergent thinking. Thinking Skills and Creativity, 22, 14-21.

Publisher's note Springer Nature remains neutral with regard to jurisdictional claims in published maps and institutional affiliations. 